\title{
Emerging Concepts for Pelvic Organ Prolapse Surgery: What is Cure?
}

\author{
Una Lee • Shlomo Raz
}

Published online: 8 December 2010

(C) The Author(s) 2010. This article is published with open access at Springerlink.com

\begin{abstract}
The objective of this review is to discuss emerging concepts in pelvic organ prolapse, in particular, "What is cure?" In a post-trial data analysis of the CARE (Colpopexy and Urinary Reduction Efforts) trial, treatment success varied tremendously depending on the definition used (19.2\%-97.2\%). Definitions that included the absence of vaginal bulge symptoms had the strongest relationships with the patients' assessment of overall improvement and treatment success. As demonstrated by this study, there are several challenges in defining cure in prolapse surgery. Additionally, the symptoms of prolapse are variable. The degree of prolapse does not correlate directly with symptoms. There are many surgical approaches to pelvic organ prolapse. Multiple ways to quantify prolapse are used. There is a lack of standardized definition of cure. The data on prolapse surgery outcomes are heterogeneous. The goal of surgical repair is to return the pelvic organs to their original anatomic positions. Ideally, we have four main goals: no anatomic prolapse, no functional symptoms, patient satisfaction, and the avoidance of complications. The impact of transvaginal mesh requires thoughtful investigation. The driving force should be patient symptoms in defining cure of prolapse.
\end{abstract}

Keywords Pelvic organ prolapse - Female pelvic floor dysfunction - Surgical outcomes · Vaginal mesh · Quality of life $\cdot$ Cystocele $\cdot$ Translabial ultrasound

\footnotetext{
U. Lee $\cdot$ S. Raz $(\square)$

Female Urology, Pelvic Medicine, and Reconstructive Surgery, Department of Urology, UCLA School of Medicine, 200 UCLA Medical Plaza, Suite 140, Los Angeles, CA 90095, USA

e-mail: sraz@mednet.ucla.edu
}

\section{Introduction}

Pelvic organ prolapse is a common condition in older women, the largest growing demographic in the United States. Pelvic prolapse affects up to $50 \%$ of women [1]. Symptomatic pelvic organ prolapse often requires surgery. Women have an $11 \%$ lifetime risk of needing surgery for pelvic floor disorders and a $29 \%$ reoperation risk for incontinence and prolapse [2]. In 1997, more than 225,000 inpatient surgical procedures for pelvic organ prolapse were undertaken in the United States, at an estimated cost of more than $\$ 1$ billion [3, 4]. The impact of pelvic organ prolapse is great on both an individual and a societal level. A great deal about this complex disease has been learned, yet there is much more to learn in this area. The objective of this article is to discuss emerging concepts in pelvic organ prolapse, in particular, "What is cure?"

\section{Defining Success in Prolapse Surgery}

In 2009, Matt Barber, in collaboration with the Pelvic Floor Disorders Network [5••], wrote, "Defining Success after Surgery for Pelvic Organ Prolapse" a post-trial data analysis of data from 322 women who completed 2-year follow-up in the CARE (Colpopexy and Urinary Reduction Efforts) trial, a randomized trial to evaluate if abdominal sacrocolpopexy with or without Burch colposuspension improves urinary stress incontinence in women without preoperative SUI. By using the pelvic organ prolapse quantification (POP-Q) assessment, the Pelvic Floor Distress Inventory responses, data on retreatment, and patients' subjective ratings of overall treatment success and global improvement, 18 different definitions of surgical success after surgery for stage 2 to 4 pelvic organ prolapse were created. 
Treatment success varied tremendously depending on the definition used (19.2\%-97.2\%), raising an important issue in the management of pelvic organ prolapse: what is cure?

No generally accepted definition for success after pelvic prolapse surgery exists. The 2001 National Institutes of Health (NIH) Workshop on Standardization of Terminology defined "optimal" anatomic outcome as stage 0 and "satisfactory" anatomic outcome as stage 1 [6]. Based on a multicenter observational study of the distribution of pelvic organ prolapse in women presenting for annual gynecologic exams, the NIH definition likely is too strict. The prevalence of pelvic organ prolapse quantification stages was $24 \%$ (stage 0 ), $38 \%$ (stage 1), $35 \%$ (stage 2 ), and $2 \%$ (stage 3 ) [6]. Of women presenting for annual exams, 75\% would not meet "optimal" definition and $40 \%$ would not meet the "satisfactory" anatomic outcome definition. According to the Barber et al. [5••] study, definitions that included the absence of vaginal bulge symptoms had the strongest relationships with the patients' assessment of overall improvement and treatment success. Additionally, definitions based on anatomic success had weak or absent correlation with patient perception of outcome [5••]. Given this finding, definitions for success after prolapse surgery should include absence of vaginal bulge symptoms.

\section{Outcomes in Prolapse Surgery}

There are four main outcomes to consider in prolapse surgery, illustrated by four overlapping circles, forming a Venn diagram (see Fig. 1):

1. Anatomy of the anterior vaginal wall, vaginal vault, posterior vaginal wall, and perineum;

2. Function of the bladder and colon, and sexual function;

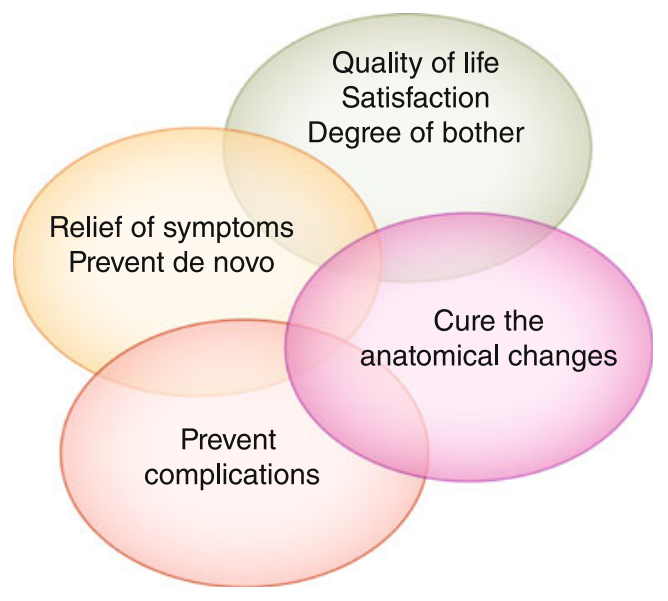

Fig. 1 Venn diagram of outcomes of prolapse surgery
3. Patient satisfaction, bother, and impact on healthrelated quality of life; and

4. Avoidance of complications.

Interestingly, the Barber [5*•] article highlights that treating anatomy is not the most important factor for patients' perception of success. Rather, the absence or presence of symptoms drives patients' perception of success. This data also confirms that there often is a disconnection between anatomy and symptoms. Vaginal prolapse within $1 \mathrm{~cm}$ from the hymen was found in $58 \%$ of women, yet $92 \%$ of women felt subjective cure. Most bowel, bladder, and pelvic symptoms thought to be associated with pelvic organ prolapse have weak to moderate correlations with the severity of prolapse [7-10]. The symptom that most correlates with advanced prolapse is the presence of a vaginal bulge that can be seen or felt $[10,11]$. Additionally, the hymen is an important anatomic cutoff point that correlates to patient symptoms of vaginal bulge $[12,13]$.

Ideally, a clinically meaningful definition of surgical success should demonstrate improved symptom-bother scores and health-related quality of life when compared to treatment failures. Vaginal bulge symptoms were found in $90 \%$ of women once the leading edge of the vagina is beyond the hymen upon straining, and patients are pleased with their surgical outcome if their vaginal bulge symptoms are eliminated [14*0]. Patient-centered goals for pelvic floor dysfunction also have been described, which broadly include four types of goals: symptom relief, activity, self-image, and general health [15].

Is the goal of prolapse surgery to produce perfect anatomy or to cure symptoms and provide better quality of life? Should we operate only for cure of the anatomic changes or only if the patient has symptoms regardless the degree of prolapse? The incidence of de novo urinary urgency after anatomic correction has been shown to be $5 \%-25 \%[16,17]$ and the incidence of de novo dyspareunia is variable, but $15 \%-25 \%$ without mesh, and as high as $63 \%$ with a mesh augmented repair [18, 19]. Surgical intervention is not without costs and complications. Therefore, the lack of standardized definitions of the disease and outcomes in pelvic organ prolapse is a critical area for our field to address.

\section{Grading and Staging of Prolapse}

Defining cure is not standardized. One contributing factor is that multiple prolapse grading and staging systems are used. The POP-Q is considered the gold standard, but has limitations. The advantages of the POP-Q are that it is quantitative, has fixed reference points, and is validated. 
The disadvantages included that it is cumbersome, variable by patient position and examination technique, and poorly utilized. Only $40 \%$ of International Continence Society/ American Urogynecologic Society members use the POP-Q in their clinical practice [20]. The two most commonly used systems in peer-reviewed urology and gynecology literature were the POP-Q (22.6\%), and the Baden-Walker (BW) system (19.8\%) [21]. The BW system is simple and well utilized, but the disadvantage is that it relates organ-specific instead of compartmental defects. Each component (urethra, cystocele, uterus, rectocele, enterocele, perineum) are graded from 0 to 4 . To address the need for a clinically relevant evaluation of prolapse grade and stage, the POP-QBaden-Walker (POPQ-BW) was developed [30]. The POPQ-BW uses the advantages of the POP-Q combined with the ease of use of the BW system. Only three simple measurements (genital hiatus, perineal body, and total vaginal length) are required. The degree of descent of each POP-Q point (Aa, Ba, C, Ap, Bp, D) is reported using the BW scale (0-4). The POPQ-BW provides a reliable, fast, reproducible evaluation of vaginal prolapse. This hybrid system perhaps may increase utilization of a grading and staging system for prolapse. The "eyeball" POP-Q, in which the measurements are estimated, also is highly correlated with the traditional POP-Q among examined patients who routinely perform the POP-Q [23].

\section{Patient-reported Global Improvement}

Women with prolapse seek treatment to improve their quality of life. Women with prolapse have decreased body image and overall quality of life [22, 24]. The Patient Global Improvement Inventory (PGII) scale is a useful tool in assessing patient improvement after surgery with one simple question, "Check the number that best describes how your post-operative condition is now, compared with how it was before you had the surgery: very much better, much better, a little better, no change, a little worse, much worse, very much worse." There was no validated global outcome assessment index in prolapse research until recently. The PGII has been validated as a measurement of global improvement after prolapse surgery [25•], and likely will be well utilized in future prolapse outcomes research.

\section{The Use of Mesh in Prolapse Surgery}

The surgical treatment of pelvic organ prolapse from a vaginal approach experienced a surge in the use of graft materials. The use of mesh in vaginal prolapse surgery stems from a desire for improved anatomic outcomes.
Interestingly, vaginal mesh kits for prolapse repair were given US Food and Drug Administration (FDA) 510(k) premarket approval by demonstrating "equivalency" to existing devices. Mesh augmented prolapse surgery demonstrates better anatomic results but has a higher risk of complications $(0 \%-23 \%)$ [26••, 27]. However, complications from vaginally placed mesh are likely underreported. The full range of complications is also difficult to quantify. Using the Clavien-Dindo Classification system [28], mesh complications that require a subsequent procedure under anesthesia falls under class IIIb. However, the degree of bother and impact on quality of life after a complication of vaginally placed mesh is difficult to fully evaluate. Mesh complications range from minor to major, and can include vaginal wall exposure, bladder and urethral mesh erosion, vaginal pain, vaginal wall induration, dyspareunia, leg pain, difficulty walking, and temporary or permanent neuropathy. Age-related changes of the vagina contribute to the development of these complications over time. An additional criticism of most mesh prolapse kits is that some do not correct vault prolapse.

\section{Use of Translabial Ultrasound}

The use of translabial ultrasound increasingly has become a helpful tool for diagnosis and preoperative planning of mesh complications [29]. Mesh is not detectable with X-ray or CT. Ultrasound is superior to MRI in detecting mesh implants. Translabial ultrasound can confirm the presence and location of residual synthetic graft material (see Fig. 2a and $b$ ). The technique involves patients in supine position with knees flexed or in lithotomy, with the bladder moderately filled. Coronal, sagittal, and axial views are obtained with the 5-9 MHz-translabial, curvilinear (Philips IU22; Philips Healthcare, Andover, MA) transducer placed on the perineum near the introitus. A dynamic evaluation is obtained with Cine image (real-time graphics on cine playback) in the resting state, squeeze, valsalva, kegel, and cough. Translabial ultrasound can assess the structure of mesh and helps to correlate clinical findings. It does not help to diagnose vaginal, bladder, or urethral erosion. Further studies on evaluating the clinical use of translabial ultrasound imaging are needed.

\section{US Food and Drug Administration Black Box Warning on Use of Mesh}

The FDA issued a black box warning on the use of mesh in October 2008, citing, "Most frequent complications included erosion through vaginal epithelium, infection, pain, urinary problems, and recurrence of prolapse and/or incontinence." The concept of learned intermediary doctrine shifts the 


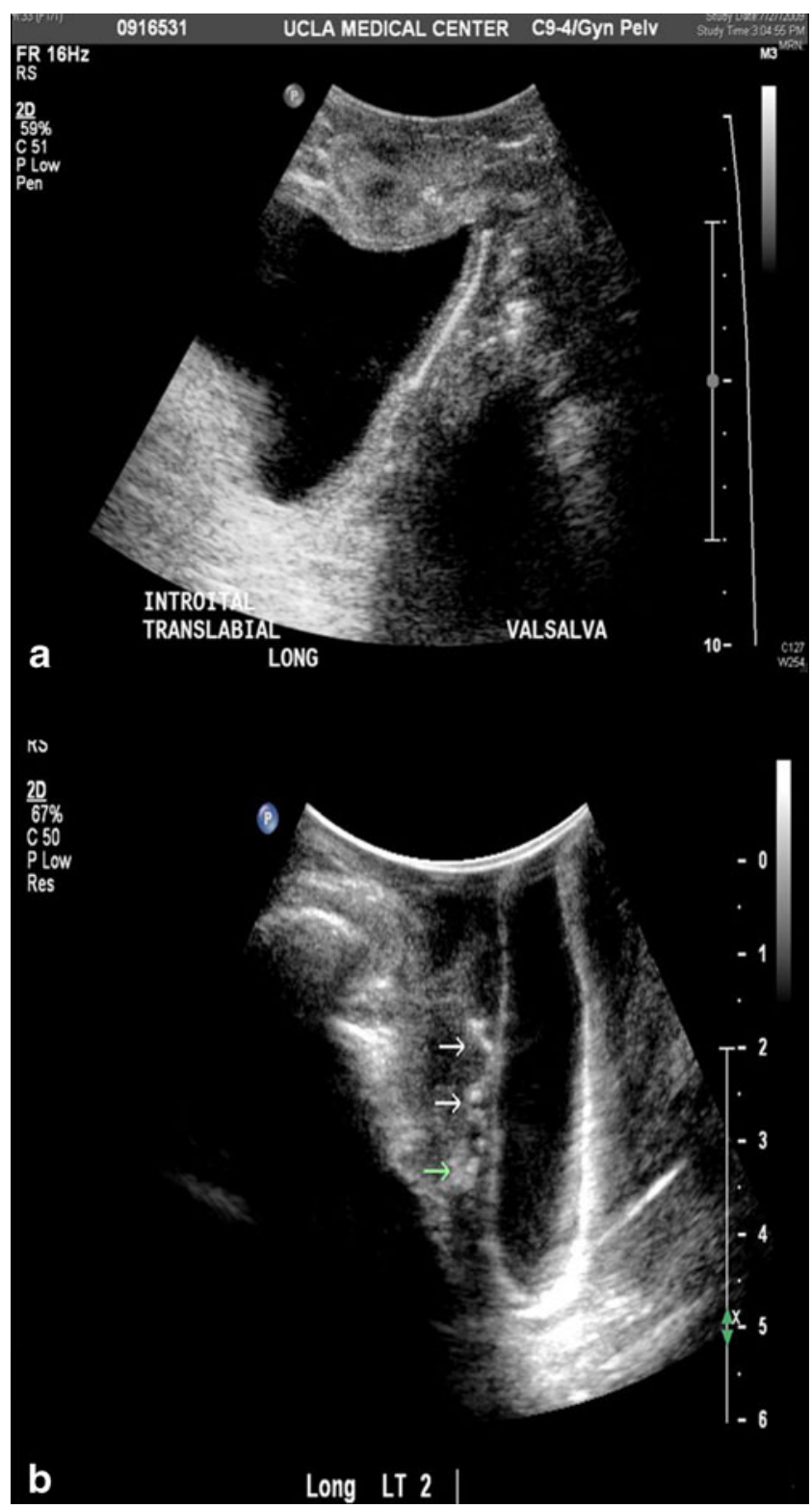

Fig. 2 a. Translabial ultrasound of anterior vaginal wall meshes in the sagittal plane. b Translabial ultrasound demonstrating folded anterior vaginal wall mesh

liability of harm away from manufacturer and onto the physician. Therefore, informed consent between physician and patient when using mesh in any female pelvic reconstructive procedure is essential.

\section{Do the Benefits of Mesh Outweigh the Risks?}

The use of mesh has demonstrated a lower risk of prolapse recurrence. However, the true impact and incidence of complications is unknown. Open, laparoscopic, or robotassisted sacrocolpopexy has a much lower risk of meshrelated complications compared to vaginal surgery. The use of mesh in vaginal prolapse repair is up to the individual surgeon and the patient. However, given the risks, the selective use of mesh in vaginal prolapse repair likely is warranted.

\section{Cystocele Repair with Interlocking Permanent Suture: an Alternative to Mesh}

Grade 4 cystocele continues to be a challenge in pelvic floor reconstruction. The traditional anterior colporrhaphy has a high recurrence rate. Mesh-augmented cystocele repairs have better anatomic results, but higher complication rates. An alternative to mesh is the use of permanent sutures to provide a net of support. A brief description of the new procedure, Cystocele repair with interlocking permanent suture is as follows: a vertical incision is made from the bladder neck to the vaginal cuff and carried out laterally. 2-0 polypropylene sutures are used to incorporate the obturator and perivesical fascia bilaterally for lateral support. Mattress sutures of 2-0 polypropylene are placed to repair the central defect. The lateral sutures then are interlocked with the central ones (see Fig. 3). The sutures then are tied, thereby reducing the cystocele and creating a supporting net. The vaginal wall is excised asymmetrically and closed as a rotational flap. Preliminary data show significant symptomatic improvement and improved quality of life. The technique has been modified to address early treatable complications, such as incidences of exposed 2 to $3 \mathrm{~mm}$ of suture, which was treated in office, and one case of ureteric obstruction, which was treated endoscopically. The "CRISP" (Cystocele Repair using Interlocking Sutures of Prolene) procedure is a promising alternative to mesh repair. Long-term data on outcomes is needed and forthcoming.

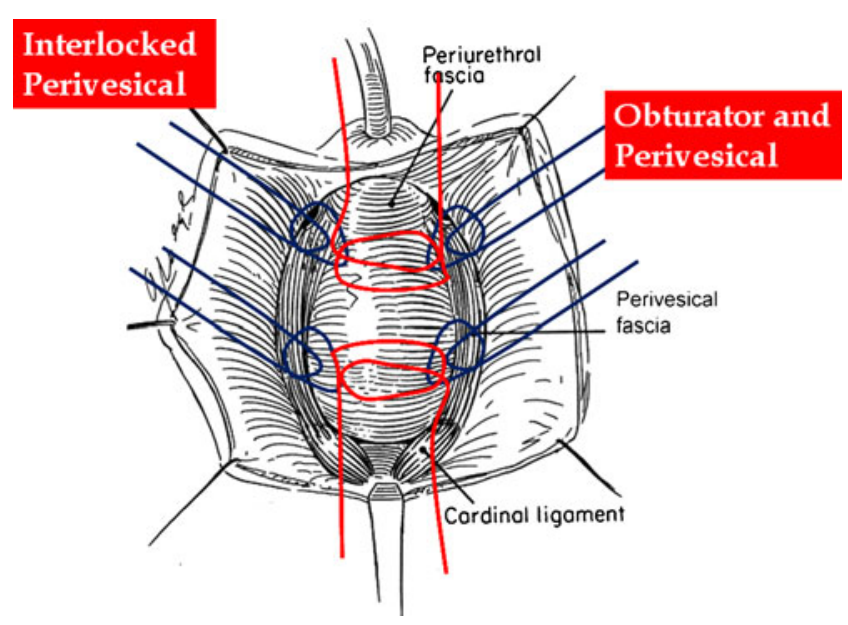

Fig. 3 Diagram of CRISP (cystocele repair using interlocking sutures of Prolene [Ethicon, Somerville, NJ]) procedure 


\section{Challenges in Curing Prolapse}

There are several challenges in defining cure in prolapse surgery. The symptoms of prolapse are variable. The degree of prolapse does not correlate directly with symptoms. There are many surgical approaches to pelvic organ prolapse. Multiple ways to quantify prolapse are used. There is a lack of standardized definition of cure. The data on prolapse surgery outcomes are heterogeneous. Therefore, the treatment of pelvic organ prolapse is challenging due in part to the lack of correlation between anatomy and symptoms, as well as to the lack of standardization of outcome measures. We try to repair the position of the pelvic organs to their original anatomic positions. Ideally, we have four main goals: no anatomic prolapse, no functional symptoms, patient satisfaction, and the avoidance of complications. What is most important is that the driving force in defining cure of prolapse should be patient symptoms.

\section{Conclusions}

Defining success after prolapse surgery requires anatomy, function, complication, and quality of life measures. The impact of transvaginal mesh requires thoughtful investigation. A thorough understanding of pelvic anatomy is required. Critical analysis of surgical outcomes is necessary.

Disclosures No potential conflicts of interest relevant to this article were reported.

Open Access This article is distributed under the terms of the Creative Commons Attribution Noncommercial License which permits any noncommercial use, distribution, and reproduction in any medium, provided the original author(s) and source are credited.

\section{References}

Papers of particular interest, published recently, have been highlighted as:

- Of importance

•. Of major importance

1. Swift S, Woodman P, O'Boyle A, et al.: Pelvic Organ Support Study (POSST): the distribution, clinical definition, and epidemiologic condition of pelvic organ support defects. Am J Obstet Gynecol. 2005;192(3):795-806.

2. Olsen AL, Smith VJ, Bergstrom JO, et al: Epidemiology of surgically managed pelvic organ prolapse and urinary incontinence. Obstet Gynecol 1997, 89(4):501-506

3. JS Brown, LE Waetjen, LL Subak, et al.: Pelvic organ prolapse surgery in the United States, 1997, Am J Obstet Gynecol 186 (2002), pp. 712-716.
4. LL Subak, LE Waetjen, S van den Eeden, et al.: Cost of pelvic organ prolapse surgery in the United States, Obstet Gynecol 98 (2001), pp. 646-651.

5. - Barber MD, Brubaker L, Nygaard I, et al.; Pelvic Floor Disorders Network: Defining success after surgery for pelvic organ prolapse. Obstet Gynecol. 2009, 114(3):600-9. This is a post-trial analysis of the CARE trial, in which 18 different definitions of success are analyzed, and treatment success varies widely based on the definition used. Absence of vaginal bulge symptoms had strongest correlation to patient perception of success.

6. Weber AM, Abrams P, Brubaker L, et al.: The standardization of terminology for researchers in female pelvic floor disorders. Int Urogynecol J Pelvic Floor Dysfunct. 2001;12(3):178-86.

7. Ellerkmann RM, Cundiff GW, Melick CF, et al.: Correlation of symptoms with location and severity of pelvic organ prolapse. Am J Obstet Gynecol. 2001;185(6):1332-7; discussion 1337-8.

8. Burrows LJ, Meyn LA, Walters MD, Weber AM. Pelvic symptoms in women with pelvic organ prolapse. Obstet Gynecol. 2004;104(5 Pt 1):982-988.

9. Ghetti C, Gregory WT, Edwards SR, et al.: Pelvic organ descent and symptoms of pelvic floor disorders. Am J Obstet Gynecol. 2005; 193(1):53-57.

10. Tan JS, Lukacz ES, Menefee SA, et al.; San Diego Pelvic Floor Consortium: Predictive value of prolapse symptoms: a large database study. Int Urogynecol J Pelvic Floor Dysfunct. 2005;16 (3):203-9; discussion 209. Epub 2004 Oct 23.

11. Bradley CS, Nygaard IE. Vaginal wall descensus and pelvic floor symptoms in older women. Obstet Gynecol. 2005;106 (4):759-66.

12. Swift SE. The distribution of pelvic organ support in a population of female subjects seen for routine gynecologic health care. Am J Obstet Gynecol. 2000;183(2):277-85.

13. Barber MD. Symptoms and outcome measures of pelvic organ prolapse. Clin Obstet Gynecol. 2005;48(3):648-61.

14. •- Swift SE, Barber MD. Pelvic Organ Prolapse: Defining the Disease. Female Pelvic Medicine and Reconstructive Surgery. 2010, 16(4), 201-203. This editorial article discusses the need to define the disease, pelvic organ prolapse, and address what is normal pelvic support; what are the symptoms that corresponds to pelvic organ prolapse and at what level of support do patients find it bothersome; and what do patients expect from treatment.

15. Hullfish KL, Bovbjerg VE, Gibson J, Steers WD. Patient-centered goals for pelvic floor dysfunction surgery: what is success, and is it achieved? Am J Obstet Gynecol. 2002;187(1):88-92.

16. de Boer TA, Kluivers KB, Withagen MI, et al.: Predictive factors for overactive bladder symptoms after pelvic organ prolapse surgery. Int Urogynecol J Pelvic Floor Dysfunct. 2010 Apr 24

17. Pham T, Kenton K, Mueller E, Brubaker L. New Pelvic Symptoms are common after reconstructive pelvic surgery. Am J Obstet Gynecol. 2009; 200(1):88.e1-5.

18. Pauls RN. Impact of gynecological surgery on female sexual function. Int J Impot Res. 2010;22(2):105-14. Epub 2010 Jan 14.

19. Carey M, Higgs P, Goh J, et al.: Vaginal repair with mesh versus colporrhaphy for prolapse: a randomised controlled trial. BJOG. 2009;116(10):1380-6. Epub 2009 Jul 7.

20. Auwad W, Freeman RM, Swift S. Is the pelvic organ prolapse quantification system (POPQ) being used? A survey of members of the International Continence Society (ICS) and the American Urogynecologic Society (AUGS). Int Urogynecol J Pelvic Floor Dysfunct. 2004;15(5):324-7. Epub 2004 May 18.

21. Muir TW, Stepp KJ, Barber MD. Adoption of the pelvic organ prolapse quantification system in peer-reviewed literature. Am J Obstet Gynecol. 2003;189(6):1632-5; discussion 1635-6.

22. Jelovsek JE, Barber MD. Women seeking treatment for advanced pelvic organ prolapse have decreased body image and quality of life. Am J Obstet Gynecol. 2006;194(5):1455-61. 
23. Karp DR, Peterson TV, Jean-Michel M, et al.: "Eyeball” POP-Q examination: shortcut or valid assessment tool? Int Urogynecol $\mathbf{J}$ Pelvic Floor Dysfunct. 2010;21(8):1005-9. Epub 2010 May 4.

24. Digesu GA, Chaliha C, Salvatore S, et al.: The relationship of vaginal prolapse severity to symptoms and quality of life. BJOG. 2005;112(7):971-6.

25. - Srikrishna S, Robinson D, Cardozo L. Validation of the Patient Global Impression of Improvement (PGI-I) for urogenital prolapse. Int Urogynecol J Pelvic Floor Dysfunct. 2010;21(5):523-8. Epub 2009 Dec 15. According to the authors, the validation of the PGII for pelvic organ prolapse will be an important tool in assessing surgical outcomes in the future.

26. • Diwadkar GB, Barber MD, Feiner B, et al.: Complication and reoperation rates after apical vaginal prolapse surgical repair: a systematic review. Obstet Gynecol. 2009;113(2 Pt 1):367-73. Review. Erratum in: Obstet Gynecol. 2009 Jun;113(6):1377. This article is an important systematic review of apical vaginal prolapse repair. Procedures were separated into three groups: traditional vaginal surgery, sacrocolpopexy, and vaginal mesh kits. The rate of complications requiring reoperation and the total reoperation rate was highest for vaginal mesh kits despite a lower reoperation rate for prolapse recurrence and shorter overall follow-up.

27. Chen CC, Ridgeway B, Paraiso MF. Biologic grafts and synthetic meshes in pelvic reconstructive surgery. Clin Obstet Gynecol. 2007;50(2):383-411.

28. Clavien PA, Barkun J, de Oliveira ML, et al.: The Clavien-Dindo classification of surgical complications: five-year experience. Ann Surg. 2009;250(2):187-96.

29. Andrea Staack, Chad Z. Baxter, Una Lee, Shelby Morrisroe, Ja-Hong Kim, Larissa Rodriguez, Shlomo Raz Impact of translabial ultrasound on diagnosis and treatment of mesh related complications. The Journal of Urology April 2010 (Vol. 183, Issue 4, Supplement, Page e647)

30. Lee U, Morrisroe S, Treat E, et al.: POPQ-BW: Combining the advantages of the Baden-Walker system and the Pelvic organ prolapse quantification (POPQ) system into one system. The Journal of Urology 2010. Vol. 183, Issue 4, Supplement, Pages e534-e535. 